\title{
Efficacy and effectiveness of COVID-19 vaccines against SARS-CoV-2 infection: interim results of a living systematic review, 1 January to 14 May 2021
}

Thomas Harder ${ }^{1}$, Judith Koch ${ }^{1}$, Sabine Vygen-Bonnet ${ }^{1}$, Wiebe Külper-Schiek ${ }^{1}$, Antonia Pilic ${ }^{1}$, Sarah Reda ${ }^{1}$, Stefan Scholz ${ }^{1}$, Ole Wichmann ${ }^{1}$

1. Robert Koch Institute, Berlin, Germany

Correspondence: Thomas Harder (hardert@rki.de)

Citation style for this article:

Harder Thomas, Koch Judith, Vygen-Bonnet Sabine, Külper-Schiek Wiebe, Pilic Antonia, Reda Sarah, Scholz Stefan, Wichmann Ole. Efficacy and effectiveness of COVID-19 vaccines against SARS-CoV-2 infection: interim results of a living systematic review, 1 January to 14 May 2021 . Euro Surveill. 2021;26(28): pii=2100563. https://doi.org/10.2807/1560-7917.ES.2021.26.28.2100563

Evidence on COVID-19 vaccine efficacy/effectiveness (VE) in preventing asymptomatic SARS-CoV-2 infections is needed to guide public health recommendations for vaccinated people. We report interim results of a living systematic review. We identified a total of 30 studies that investigated VE against symptomatic and/ or asymptomatic infection. In fully vaccinated individuals, VE against symptomatic and asymptomatic infections was $80-90 \%$ in nearly all studies. Fully vaccinated persons are less likely to become infected and contribute to transmission.

Vaccination against severe acute respiratory syndrome coronavirus 2 (SARS-CoV-2) infection plays a key role in the containment of the coronavirus disease (COVID19) pandemic. All vaccines approved by the European Medical Agency (EMA) at the time of writing demonstrated high vaccine efficacy/effectiveness (VE) against severe COVID-19. With vaccination programmes being implemented in most European countries, it becomes urgent to assess the extent to which these vaccines are also able to prevent symptomatic and asymptomatic infections to guide public health recommendations and develop strategies for fully vaccinated people.

In December 2020, the Robert Koch Institute (RKI), in collaboration with the National Immunisation Technical Advisory Groups (NITAGs) network coordinated by the European Centre for Disease Prevention and Control (ECDC) initiated a living systematic review on the VE and safety of European Union (EU)-licensed COVID-19 vaccines (PROSPERO registration: CRD42020208935). In this paper, only efficacy and effectiveness data but not those on safety will be covered. In detail, we report the interim results of the review focusing on two research questions:

What is the efficacy/effectiveness of COVID-19 vaccines in preventing SARS-CoV-2 infections (irrespective of whether those infected were symptomatic or asymptomatic)?

What is the efficacy/effectiveness of COVID-19 vaccines in preventing asymptomatic SARS-CoV-2 infections?

\section{Literature search}

This living systematic review follows the recommendations of the Preferred Reporting Items for Systematic Review and Meta-Analysis (PRISMA) guideline (Supplement Part S1). Monthly searches were done and results were immediately incorporated into the evidence base. We considered studies of any design as long as they had a comparison group that investigated VE against SARS-CoV-2 infection and/or asymptomatic SARS-CoV-2 infection after vaccination with an EMA-approved COVID-19 vaccine (see Supplement Part S2 for complete population intervention comparison outcomes (PICO) questions). No restrictions were made regarding publication language or status. The review started on 1 January 2021. The end date of this interim analysis was 14 May 2021.

We used an internal COVID-19 literature database constructed by the RKI library to search for relevant studies. This database covers PubMed, Embase (including Medline) and the preprint servers ArRvix, BioRxiv, ChemRxiv, MedRxiv, Preprints.org, ResearchSquare and Social Science Research Network (SSRN) (see Supplement Part S3 for search strategy). In addition, we hand-searched the websites of the ECDC, the United States (US) Centers for Disease Control and Prevention, the Public Health Agency of Canada and Public Health England for additional studies and reports. Potentially relevant publications were screened at title/abstract and full-text level by two independent investigators for eligibility. Disagreements were resolved by discussion. From the identified studies, data were extracted as described in the PROSPERO protocol and summarised 


\section{FIGURE}

PRISMA flowchart of the living systematic review on efficacy and effectiveness of COVID-19 vaccines against SARS-CoV-2 infection

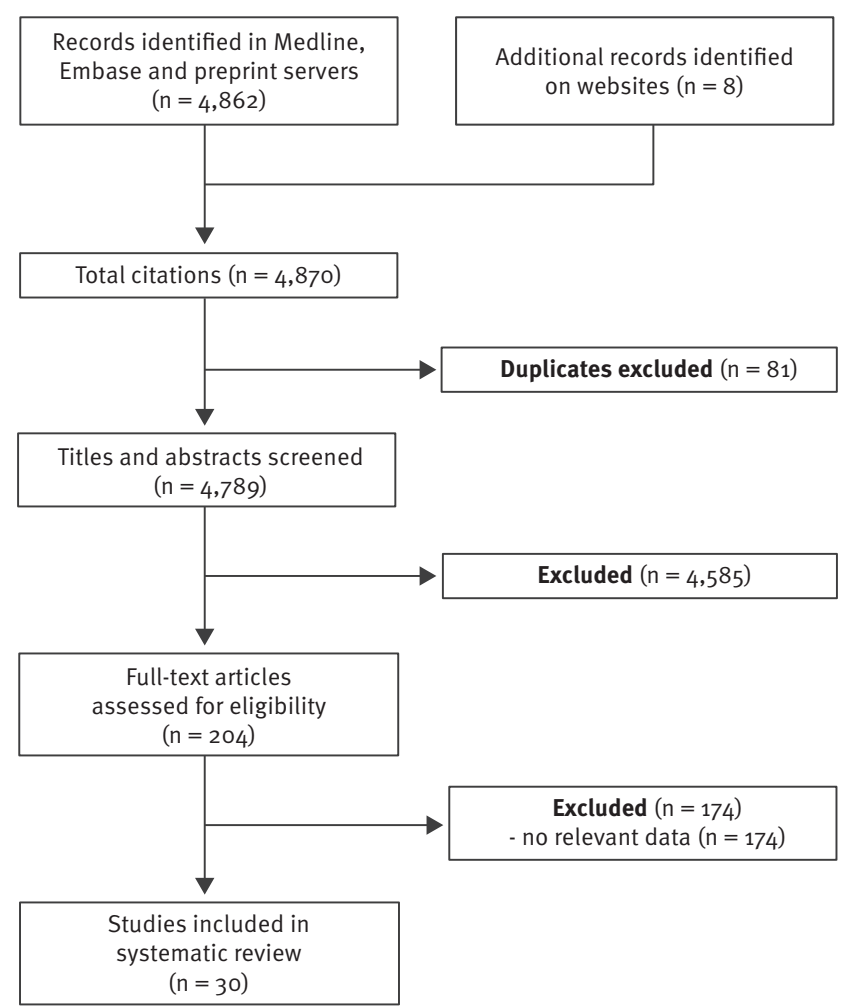

COVID-19: coronavirus disease; SARS-CoV-2: severe acute respiratory syndrome coronavirus 2; PRISMA: Preferred Reporting Items for Systematic Review and Meta-Analysis.

Date of last search: 14 May 2021.

in tabular form. For randomised controlled trials (RCTs), risk of bias was assessed using the Cochrane Risk of Bias tool-2 (RoB-2) [1]. To non-randomised studies, ROBINS-I was applied [2].

Due to heterogeneity of study design, time point of analysis, vaccine used, population and settings, we did not perform a meta-analysis.

\section{Study screening}

By 14 May 2021 (the date of last search), we identified and screened a total of 4,870 entries. After screening 204 full-text articles, 30 studies were included (Figure) [3-32]. Twenty-six studies reported on infections (irrespective of whether they were symptomatic or not), including three studies that reported both outcomes, and four additional studies reported exclusively on asymptomatic infections.

\section{Prevention of infection}

Of the 30 studies, 26 investigated the efficacy/effectiveness of COVID-19 vaccines in preventing SARS-CoV-2 infections, based on reports of number of symptomatic and asymptomatic PCR-positive individuals
[3-18,22-31] (Table 1). Studies were conducted in eight different countries (Denmark $(n=1)$, Israel $(n=4)$, Italy $(n=1)$, Qatar $(n=1)$, Spain $(n=2)$, Sweden $(n=1)$, UK $(n=8)$, US $(n=7))$ and one study was multi-centric [22]. They included between 463 and 2,183,000 participants aged 16-99 years. Two studies were RCTs, 19 studies had a cohort design and five were case-control studies, including two with test-negative design. In 12 studies, the effectiveness of Comirnaty (BionTech, Mainz, Germany/Pfizer, Puurs, Belgium) was evaluated. Two studies investigated COVID-19 Vaccine Janssen (Janssen-Cilag International, Beerse, Belgium), one study studied Vaxzevria (AstraZeneca/Oxford, Oxford, United Kingdom (UK)), and 11 studies investigated more than one vaccine.

One-dose efficacy/effectiveness was investigated in 24 studies (Table 1) and estimates ranged from $16.9 \%$ to $91.2 \%$, with the majority of estimates ranging between $60 \%$ and $70 \%$. The VE was lower in older (e.g. longterm care facility inhabitants) than in younger participants (e.g. healthcare workers). However, age-related effects could not be assessed in a number of studies since subgroup data were not reported. Vaccine type and study design did not appear to have an impact on VE estimates.

In 17 of 26 studies, VE was reported after the second dose. Estimates ranged between $61.7 \%$ and $98.6 \%$. One study found an incidence reduction of $99 \%$ [10]. The majority of estimates ranged from $80 \%$ to $90 \%$. Again, VE estimates were not affected by participant age, vaccine type and/or study design (see Supplement Part $\mathrm{S}_{4}$ for exact definitions of outcomes).

\section{Prevention of asymptomatic infection}

Seven of 30 studies investigated VE against asymptomatic SARS-CoV-2 infections [11,19-22,31,32] (Table 2). With the exception of one study that had a multicentre design with study centres in the US, Brazil and South Africa, the remainder were performed in single centres in Israel $(n=3)$, the UK $(n=1)$ and the US $(n=2)$. Studies included between 5,217 and more than 300,000 participants, with three of them including healthcare workers only. Only the multicentre study investigating COVID19 vaccine Janssen was an RCT [22]. The other studies had a cohort design and investigated Comirnaty or Comirnaty and COVID-19 vaccine Moderna using either hospital, insurance or surveillance data. In five of these six studies, VE against asymptomatic infection after one dose of Comirnaty or COVID-19 vaccine Moderna ranged from $36 \%$ to $79 \%$. Five cohort studies also analysed VE against asymptomatic infection after a second dose and reported VE estimates between $80 \%$ and $94 \%$. For the single-dose regimen of COVID-19 vaccine Janssen, VE against asymptomatic infections was $74 \%$ in the RCT [22].

\section{Risk of bias}

Risk of bias was low in one RCT [22]. In the other RCT [8], we detected some concerns due to different 


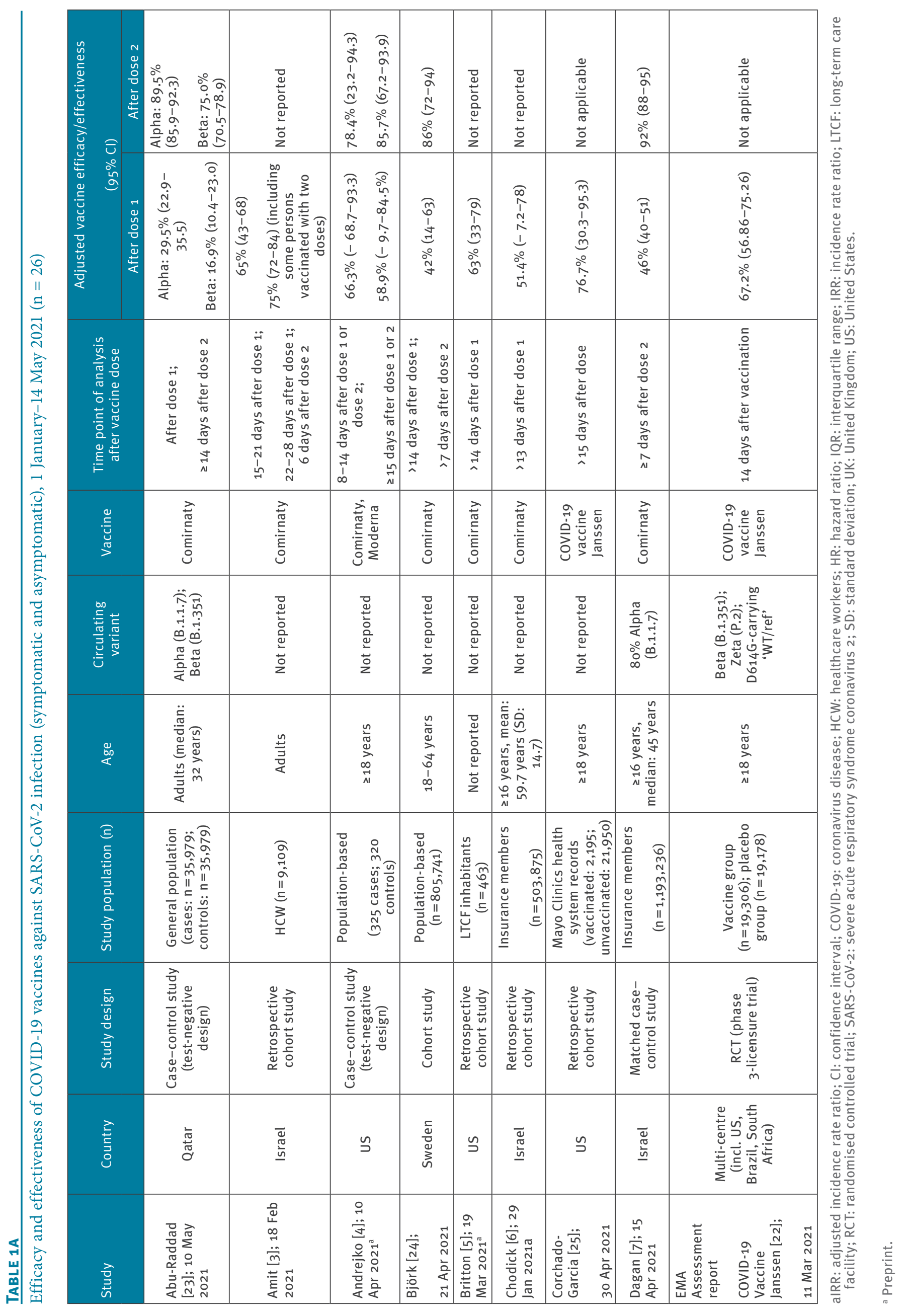









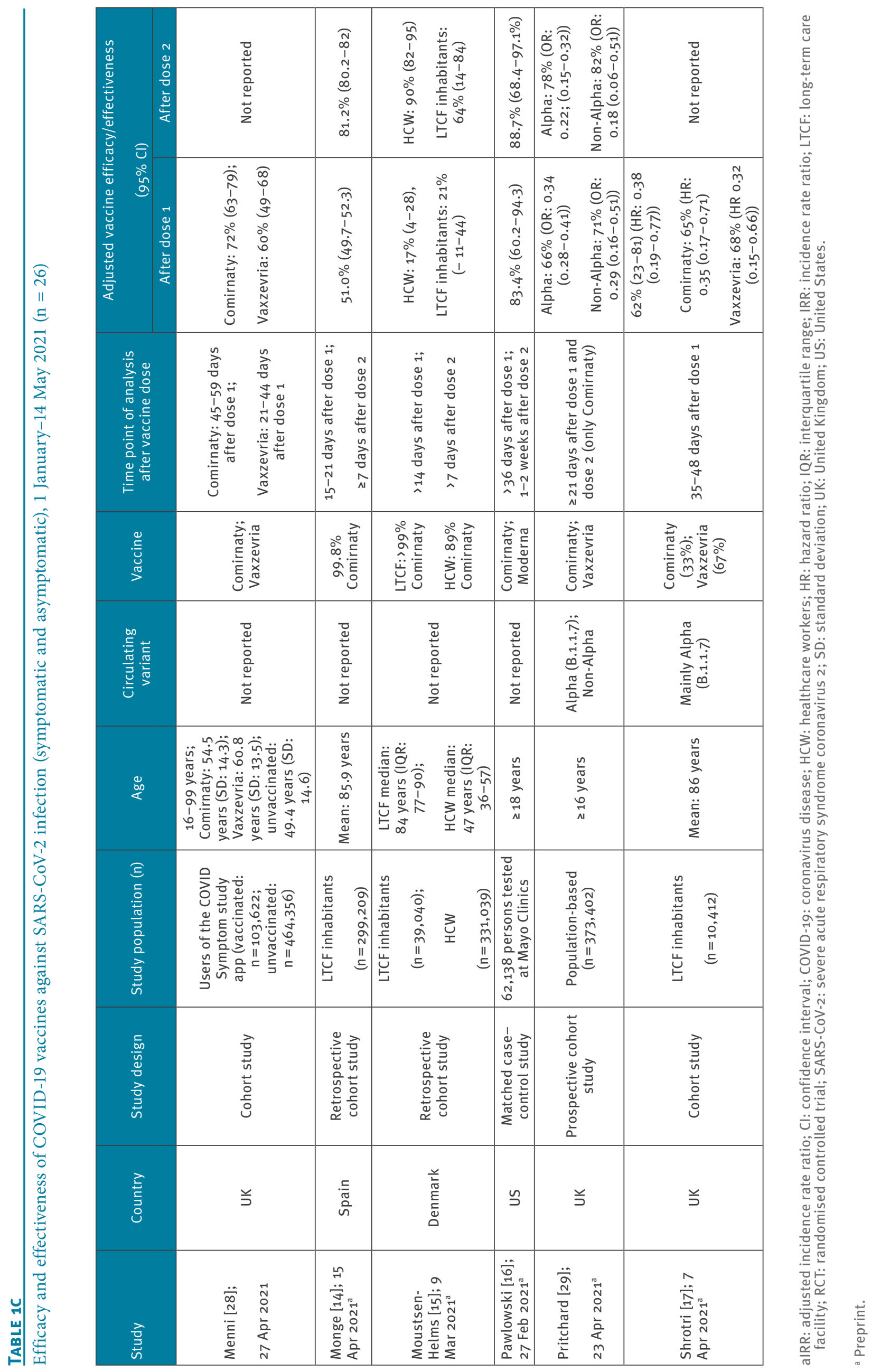




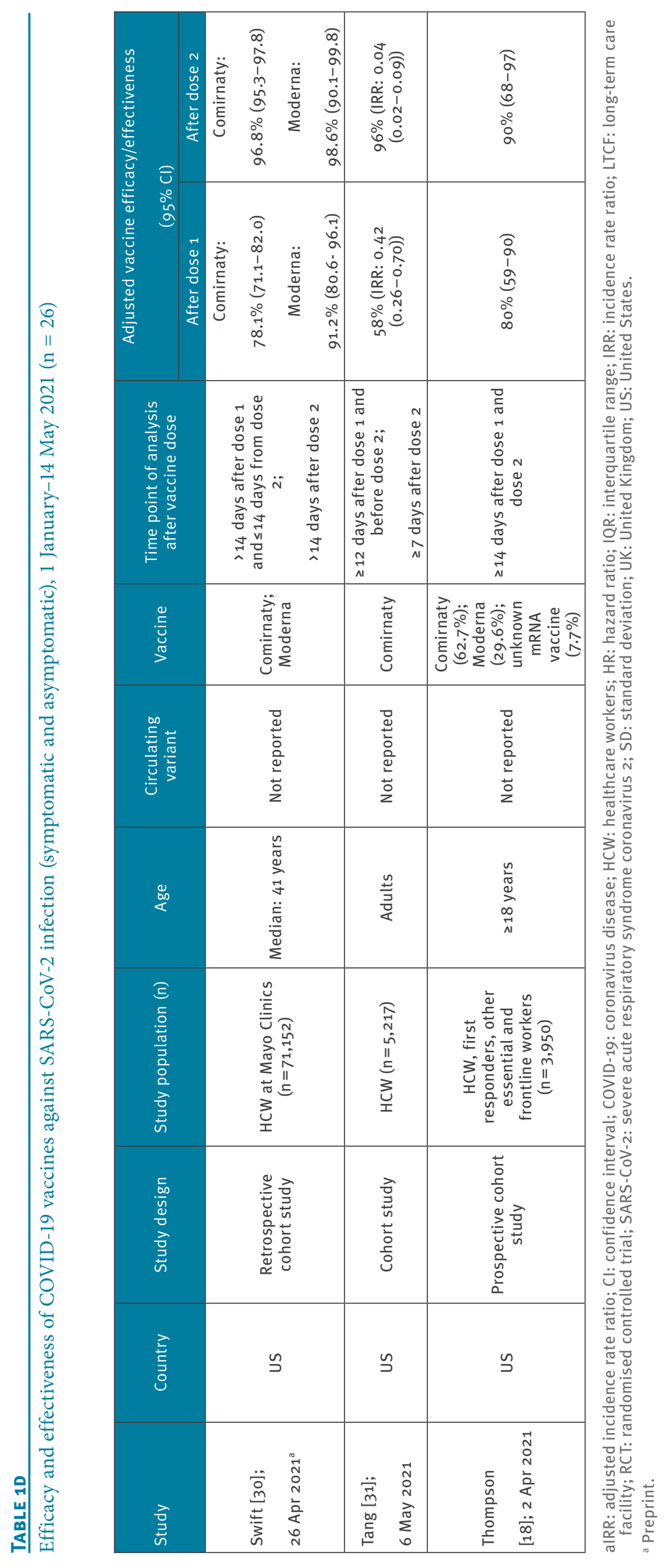




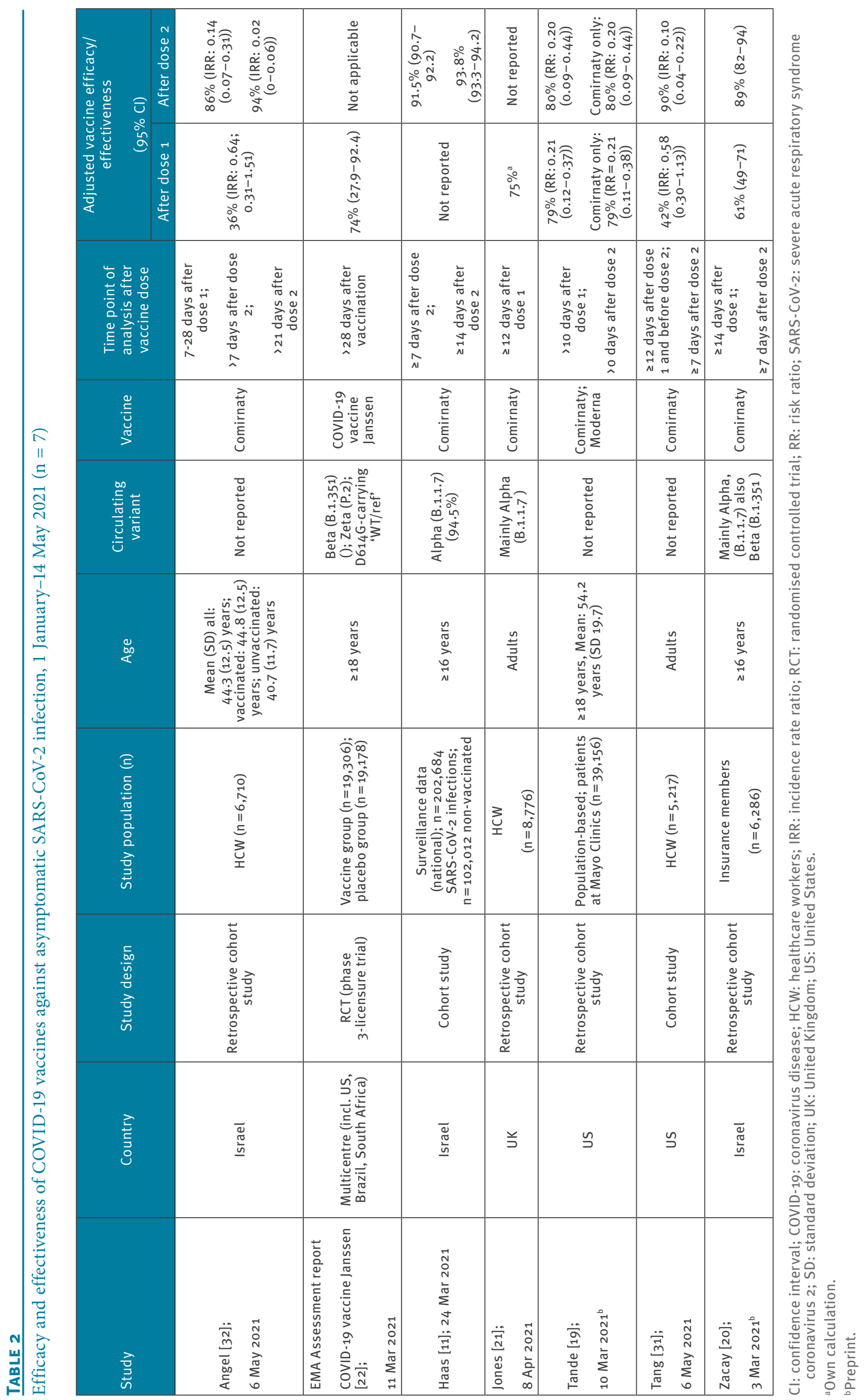


vaccine dosages being analysed. Of the 28 non-randomised studies, risk of bias was critical in four studies which did not adjust for confounders and reported unadjusted estimates. In a further two non-randomised studies, risk of bias was considered to be serious because adjustment for confounders was inappropriate. Besides one study with unclear risk of bias, all remaining studies had moderate risk of bias, mainly due to possible residual confounding (see Supplement Part S $_{5}$ for details).

\section{Discussion}

These interim results of a living systematic review show that after completed course the EMA-approved COVID-19 vaccines have a VE of $80 \%$ to $90 \%$ in preventing SARS-CoV2 infections, including asymptomatic ones. We found some indication that VE estimates are not reduced in cases infected with variant of concern (VOC) Alpha (Phylogenetic Assignment of Named Global Outbreak (Pango) lineage designation B.1.1.7), however these results should be interpreted with caution. VE against infection can also be regarded as an indicator of how well a vaccine prevents transmission. In addition, studies suggest that persons who become SARS-CoV-2-positive despite vaccination had a shorter duration of virus shedding and lower viral load [8].

Some of our methodological limitations stem from the rapidly changing publication landscape of COVID-19 vaccine studies. In particular, non-randomised studies on real-world effectiveness are continuously and frequently published on preprint servers. Although we systematically searched seven preprint servers, additional studies could have been published on other servers or websites that we did not capture. Moreover, it has to be considered that these studies did not undergo peer review and should therefore be considered with caution. A recent study reported that some final journal publications of COVID-19 studies differ to a certain extent from versions which were previously published on pre-print servers [33]. A further limitation is the small number of countries where the studies were performed, including the possibility that some studies, in particular those from Israel and the US, might have analysed partly overlapping study populations. The majority of studies included were conducted in persons vaccinated with Comirnaty. At the time point of data cut for this interim analysis, only limited information was available on VOCs other than Alpha. Meanwhile, some studies have been published indicating reduced effectiveness against infections with VOC Delta for Comirnaty and Vaxzevria, whereas effectiveness against hospitalisation was unchanged, as compared to VOC Alpha [34,35].

\section{Conclusion}

Results of this living systematic review imply that COVID-19 vaccines are highly effective in preventing SARS-CoV-2 infections, including those which are asymptomatic. From a public health perspective, it can be concluded that fully vaccinated persons might in some instances still become PCR-positive for SARSCoV-2 but only play a minor role in the transmission of SARS-COV-2.

\section{Acknowledgements}

The authors would like to thank the members of the EU/EEA NITAG Collaboration Working Group 3 for valuable comments on the PICO questions and review protocol.

\section{Conflict of interest}

None declared.

Authors' contributions

TH conceived the study, was second reviewer, and drafted the manuscript. JK and SVB were primary reviewers and contributed to the manuscript. SR, WKS and AP were second reviewers and contributed to the manuscript. SS provided input into the interpretation of the results. OW held general oversight of the conducted work and revised the manuscript. All authors contributed to the interpretation of the data and provided important intellectual content to the manuscript.

\section{References}

1. Sterne JAC, Savović J, Page MJ, Elbers RG, Blencowe NS, Boutron I, et al. RoB 2: a revised tool for assessing risk of bias in randomised trials. BMJ. 2019;366:14898. https://doi. org/10.1136/bmj.l4898 PMID: 31462531

2. Sterne JA, Hernán MA, Reeves BC, Savović J, Berkman ND, Viswanathan M, et al. ROBINS-I: a tool for assessing risk of bias in non-randomised studies of interventions. BM]. 2016;355:i4919. https://doi.org/10.1136/bmj.i4919 PMID: 27733354

3. Amit S, Regev-Yochay G, Afek A, Kreiss Y, Leshem E. Early rate reductions of SARS-CoV-2 infection and COVID-19 in BNT162b2 vaccine recipients. Lancet. 2021;397(10277):875-7. https://doi. org/10.1016/S0140-6736(21)00448-7 PMID: 33610193

4. Andrejko K, Pry J, Myers JF, Jewell NP, Openshaw J, Watt J, et al. Early evidence of COVID-19 vaccine effectiveness within the general population of California. medRxiv. 2021.04.08.21255135. https://doi.org/10.1101/2021.04.08.21 255135

5. Britton A, Jacobs Slifka KM, Edens C, Nanduri SA, Bart SM, Shang N, et al. Effectiveness of the Pfizer-BioNTech COVID-19 vaccine among residents of two skilled nursing facilities experiencing COVID-19 outbreaks-Connecticut, December 2020-February 2021. MMWR Morb Mortal Wkly Rep. 2021;70(11):396-401. https://doi.org/10.15585/mmwr. mm7011e3 PMID: 33735160

6. Chodick G, Tene L, Patalon T, Gazit S, Tov AB, Cohen D, et al. The effectiveness of the first dose of $\mathrm{BNT}_{1} \mathrm{C}_{2} \mathrm{~b}_{2}$ vaccine in reducing SARS-CoV-2 infection 13-24 days after immunization: real-world evidence. medRxiv. 2021:2021.01.27.21250612. https://doi.org/10.2139/ssrn.3769977

7. Dagan N, Barda N, Kepten E, Miron O, Perchik S, Katz MA, et al. BNT162 b2 mRNA Covid-19 vaccine in a nationwide mass vaccination setting. N Engl J Med. 2021;384(15):1412-23. https://doi.org/10.1056/NEJMoa2101765 PMID: 33626250

8. Emary KRW, Golubchik T, Aley PK, Ariani CV, Angus B, Bibi $\mathrm{S}$, et al. Efficacy of ChAdOx1 nCoV-19 (AZD1222) vaccine against SARS-CoV-2 variant of concern 202012/01 (B.1.1.7): an exploratory analysis of a randomised controlled trial. Lancet. 2021;397(10282):1351-62. https://doi.org/10.1016/S01406736(21)00628-0 PMID: 33798499

9. Glampson B, Brittain J, Kaura A, Mulla A, Mercuri L, Brett S, et al. North West London Covid-19 vaccination programme: realworld evidence for vaccine uptake and effectiveness. medRxiv. 2021.04.08.21254580. https://doi.org/10.1101/2021.04.08.21 254580

10. Guijarro C, Galán I, Martínez-Ponce D, Pérez-Fernández $E$, José Goyanes M, Castilla V, et al. Dramatic drop of new SARS-CoV-2 infections among health care workers after the first dose of the BNT162b2 mRNA Covid-19 vaccine. medRxiv. 
2021.03.24.21254238. https://doi.org/10.1101/2021.03.24.21 254238

11. Haas EJ, Angulo FJ, McLaughlin JM, Anis E, Singer SR, Khan F, et al. Nationwide vaccination campaign with BNT162 b2 in Israel demonstrates high vaccine effectiveness and marked declines in incidence of SARS-CoV-2 infections and COVID-19 cases, hospitalizations, and deaths. SSRN. 2021. Available from: https://ssrn.com/abstract $=3811387$

12. Hall VJ, Foulkes S, Saei A, Andrews N, Oguti B, Charlett A, et al. Effectiveness of BNT162 b2 mRNA vaccine against infection and COVID-19 vaccine coverage in healthcare workers in England, multicentre prospective cohort study (the SIREN Study). SSRN. 2021. Available from: https://papers.ssrn.com/sol3/papers. cfm?abstract_id=3790399

13. Lumley SF, Rodger G, Constantinides B, Sanderson N, Chau KK, Street TL, et al. An observational cohort study on the incidence of SARS-CoV-2 infection and B.1.1.7 variant infection in healthcare workers by antibody and vaccination status. medRxiv. 2021.03.09.21253218. https://doi.org/10.1101/2021. 03.09 .21253218

14. Monge S, Olmedo C, Alejos B, Lapeña MF, Sierra MJ, Limia A. Direct and indirect effectiveness of mRNA vaccination against SARS-CoV-2 infection in long-term care facilities in Spain. medRxiv. 2021.04.08.21255055. https://doi.org/10.1101/2021. 04.08 .21255055

15. Moustsen-Helms IR, Emborg H-D, Nielsen J, Nielsen KF, Krause TG, Molbak K, et al. Vaccine effectiveness after 1 st and 2nd dose of the BNT162b2 mRNA Covid-19 vaccine in long-term care facility residents and healthcare workers-a Danish cohort study. MedRxiv. 2021.03.08.21252200. https://doi.org/10.1101 /2021.03.08.21252200

16. Pawlowski C, Lenehan P, Puranik A, Agarwal V, Venkatakrishnan A, Niesen MJ, et al. FDA-authorized COVID-19 vaccines are effective per real-world evidence synthesized across a multi-state health system. MedRxiv. 2021.02.15.21251623. https://doi.org/10.1101/2021.02.15.212 51623

17. Shrotri M, Krutikov M, Palmer T, Giddings R, Azmi B, Subbarao $S$, et al. Vaccine effectiveness of the first dose of ChAdOx1 nCoV-19 and BNT162 b2 against SARS-CoV-2 infection in residents of long-term care facilities in England (VIVALDI): a prospective cohort study. Lancet Infect Dis. 2021;S14733099(21)00289-9 PMID: 34174193

18. Thompson MG, Burgess JL, Naleway AL, Tyner HL, Yoon SK, Meece J, et al. Interim estimates of vaccine effectiveness of BNT162b2 and mRNA-1273 COVID-19 vaccines in preventing SARS-CoV-2 infection among health care personnel, first responders, and other essential and frontline workers eight U.S. locations, December 2020-March 2021. MMWR Morb Mortal Wkly Rep. 2021;70(13):495-500. https://doi. org/10.15585/mmwr.mm7013e3 PMID: 33793460

19. Tande AJ, Pollock BD, Shah ND, Farrugia G, Virk A, Swift $M$, et al. Impact of the COVID-19 vaccine on asymptomatic infection among patients undergoing pre-procedural COVID-19. molecular screening. Clin Infect Dis. 2021; ciab229. https://doi. org/10.1093/cid/ciab229 PMID: 33704435

20. Heymann AD, Zacay G, Shasha D, Bareket R, Kadim I, Sikron $\mathrm{FH}$, et al. BNT162 b2 vaccine effectiveness in preventing asymptomatic infection with SARS-CoV-2 virus: a nationwide historical cohort study. SSRN. 2021. Available from: https:// ssrn.com/abstract $=3796868$

21. Jones NK, Rivett L, Seaman S, Samworth RJ, Warne B, Workman $C$, et al. Single-dose BNT162 b2 vaccine protects against asymptomatic SARS-CoV-2 infection. eLife. 2021;10:10:e68808. https://doi.org/10.7554/eLife.68808 PMID: 33830018

22. European Medicines Agency (EMA). Assessment report COVID-19 Vaccine Janssen. Amsterdam: EMA. 2021. Available from: https://www.ema.europa.eu/en/documents/assessmentreport/covid-19-vaccine-janssen-epar-public-assessmentreport_en.pdf

23. Abu-Raddad LJ, Chemaitelly H, Butt AA, National Study Group for COVID-19 Vaccination. Effectiveness of the BNT162 b2 Covid-19 vaccine against the B.1.1.7 and B.1.351 variants. N Engl J Med. 2021;385(2):187-9. https://doi.org/10.1056/ NEJMC2104974 PMID: 33951357

24. Bjork J, Inghammar M, Moghaddassi M, Rasmussen M, Malmqvist U, Kahn F. Effectiveness of the BNT162 b2 vaccine in preventing COVID-19 in the working age population-first results from a cohort study in southern Sweden. medRxiv. 2021.04.20.21254636. https://doi.org/10.1101/2021.04.20.21 254636

25. Corchado-Garcia J, Hughes T, Cristea-Platon T, Lenehan P, Pawlowski C, Bade S, et al. Real-world effectiveness of Ad26. COV2. S adenoviral vector vaccine for COVID-19. SSRN. 2021. Available from: https://ssrn.com/abstract $=3835737$
26. Fabiani M, Ramigni M, Gobbetto V, Mateo-Urdiales A, Pezzotti $P$, Piovesan C. Effectiveness of the Comirnaty (BNT162 b2, BioNTech/Pfizer) vaccine in preventing SARS-CoV-2 infection among healthcare workers, Treviso province, Veneto region, Italy, 27 December 2020 to 24 March 2021. Euro Surveill. 2021;26(17):2100420. https://doi.org/10.2807/1560-7917. ES.2021.26.17.2100420 PMID: 33928898

27. Mason TF, Whitston M, Hodgson J, Watkinson RE, Lau Y-S, Abdulrazeg 0, et al. Effects of BNT162b2 mRNA vaccine on Covid-19 infection and hospitalisation among older people: matched case control study for England. medRxiv. 2021. 04.19.21255461. https://doi.org/10.1101/2021.04.19.21255461

28. Menni C, Klaser K, May A, Polidori L, Capdevila J, Louca P, et al. Vaccine side-effects and SARS-CoV-2 infection after vaccination in users of the COVID Symptom Study app in the UK: a prospective observational study. Lancet Infect Dis. 2021;21(7):939-49. https://doi.org/10.1016/S14733099(21)00224-3 PMID: 33930320

29. Pritchard E, Matthews PC, Stoesser N, Eyre DW, Gethings O, Vihta K-D, et al. Impact of vaccination on SARS-CoV-2 cases in the community: a population-based study using the UK's COVID-19 infection survey. medRxiv. 2021.04.22.21255913. https://doi.org/10.1101/2021.04.22.21255913

30. Swift MD, Breeher LE, Tande AJ, Tommaso CP, Hainy CM, Chu H, et al. Effectiveness of mRNA COVID-19 vaccines against SARSCoV-2 infection in a cohort of healthcare personnel. Clin Infect Dis. 2021; ciab361. https://doi.org/10.1093/cid/ciab361 PMID: 33900384

31. Tang L, Hijano DR, Gaur AH, Geiger TL, Neufeld EJ, Hoffman JM, et al. Asymptomatic and symptomatic SARS-CoV-2 infections after BNT162 b2 vaccination in a routinely screened workforce. JAMA. 2021;325(24):2500-2. https://doi.org/10.1001/ jama.2021.6564 PMID: 33956050

32. Angel Y, Spitzer A, Henig O, Saiag E, Sprecher E, Padova $\mathrm{H}$, et al. Association between vaccination with BNT162 b2 and incidence of symptomatic and asymptomatic SARSCoV-2 infections among health care workers. JAMA. 2021;325(24):2457-65. https://doi.org/10.1001/jama.2021.7152 PMID: 33956048

33. Bero L, Lawrence R, Leslie L, Chiu K, McDonald S, Page M, et al. Comparison of preprints and final journal publications from COVID-19 studies: discrepancies in results reporting and spin in interpretation. medRxiv. 2021. 04.12.21255329. https://doi. org/10.1101/2021.04.12.21255329

34. Sheikh A, McMenamin J, Taylor B, Robertson C, Public Health Scotland and the EAVE II Collaborators. SARS-CoV-2 Delta VOC in Scotland: demographics, risk of hospital admission, and vaccine effectiveness. Lancet. 2021;397(10293):24612462. https://doi.org/10.1016/S0140-6736(21)01358-1 PMID: 34139198

35. Stowe J, Andrews N, Gower C, Gallagher E, Utsi L, Simmons R. Effectiveness of COVID-19 vaccines against hospital admission with the Delta (B. 1.617. 2) variant. Public Health England. 2021. Available from: https://media.tghn.org/articles/ Effectiveness_of_COVID-19_vaccines_against_hospital_ admission_with_the_Delta_B._G6gnnqJ.pdf

\section{License, supplementary material and copyright}

This is an open-access article distributed under the terms of the Creative Commons Attribution (CC BY 4.0) Licence. You may share and adapt the material, but must give appropriate credit to the source, provide a link to the licence and indicate if changes were made.

Any supplementary material referenced in the article can be found in the online version.

This article is copyright of the authors or their affiliated institutions, 2021. 